\title{
Detailed Study of AlAs-Oxidized Apertures in VCSEL Cavities for Optimized Modal Performance
}

\author{
B. Demeulenaere, P. Bienstman, B. Dhoedt, and R. G. Baets, Senior Member, IEEE
}

\begin{abstract}
We present a numerical optical model for calculating threshold material gain in vertical-cavity surface-emitting lasers. It is based on a vectorial solution of Maxwell's equations and therefore gives exact results where other approaches fail, e.g., in the case of oxide-confined devices, which have high lateral index contrasts. Results are given concerning the influence of oxide window thickness and position on threshold gain and modal stability. We also propose an intuitive plane-wave model to enhance the physical understanding of these effects.
\end{abstract}

Index Terms-Distributed Bragg reflector lasers, laser modes, semiconductor device modeling, semiconductor lasers, surfaceemitting lasers.

\section{INTRODUCTION}

$\mathbf{V}$ ERTICAL-CAVITY surface-emitting lasers (VCSEL's) have been the topic of intense research for several years now. In particular, since the introduction of the controlled oxidation of semiconductor material with high Alcontent, short-wavelength VCSEL's operating between 850 and $980 \mathrm{~nm}$ have experienced a tremendous improvement of their operation characteristics. An oxide aperture acts as a current window, enabling the active region to be pumped more efficiently. Apart from this electrical property, oxide apertures can also have beneficial optical effects, counteracting diffraction and improving the modal stability of oxide-confined VCSEL's. In many respects, these devices now outperform traditional edge-emitting lasers. Oxide-confined VCSEL's have been demonstrated with extremely low threshold currents [1], [2] and record high wall plug efficiencies [3]. Low operating voltages [4] as well as very high fabrication yields [5] have been reported. These milestones, together with the inherent possibility of wafer scale testing, easy packaging, and very good output beam quality make VCSEL's the ideal light source for short-haul optical communication and for optical interconnect [6].

In the past, the optical properties of the AlAs-oxide aperture have been looked upon as a position-dependent cavity resonance [7], a position-dependent phase shift [8], or as an

Manuscript received July 31, 1998; revised November 9, 1998. This work was supported in part by the European ACTS-024 Project (VERTICAL). The work of B. Demeulenaere and P. Bienstman was supported by the Flemish National Fund for Scientific Research (FWO-Vlaanderen) through doctoral fellowships. The work of B. Dhoedt was supported by the Flemish Institute for Science and Technology in Industry (IWT) through a post-doctoral fellowship.

B. Demeulenaere was with the Department of Information Technology, University of Gent-IMEC, B-9000 Gent, Belgium. He is now with Barco R\&D Department, B-8500 Kortrijk, Belgium.

P. Bienstman, B. Dhoedt, and R. G. Baets are with the Department of Information Technology, University of Gent-IMEC, B-9000 Gent, Belgium.

Publisher Item Identifier S 0018-9197(99)01725-X. intracavity lens counteracting diffraction [9]. All these points of view are based on simple and approximate models, aimed at an intuitive understanding of oxide windows.

Numerical methods based on scalar wave equations are presented in [10] and [11]. However, for structures with dimensions on the order of the wavelength and where strong index contrasts are present (e.g., oxide-apertured devices), a vectorial approach seems to be more appropriate, especially for the study of polarization properties of higher order transverse modes.

Some work has been done already in developing vectorial optical models for VCSEL structures. In [12], an approach is presented based on the weighted index method, but is unable to handle diffraction effects which become predominant when dealing with small devices. Similar methods are outlined in [13] and [14] to calculate the mode spectrum of a cold cavity, based on expansion into hybrid modes of optical fibers. However, no optical gain or absorption is taken into account in these models, so the threshold gain cannot be evaluated directly. It is the aim of this paper to present a full optical laser model, based on the vectorial solution of Maxwell's equations, that is able to handle diffraction effects and that can calculate threshold material gain directly. Our approach also differs from that of [13] and [14] in the sense that we enclose the structure under study in a metallic cylinder and use the discrete set of modes of this cylinder to express all the fields. In contrast, the field is expanded in [13] and [14] in hybrid modes of an open optical fiber waveguide, where one has to deal with a continuum of evanescent modes.

Using our model, results will be presented on the influence of oxide windows on threshold material gain and modal stability. Even though our model is not self-consistent-electrical and thermal effects are not taken into account explicitly- the results give a good qualitative idea of the different structures with respect to modal stability. Finally, to enhance the understanding of the data, we will discuss an intuitive plane-wave model for oxide apertures.

\section{Description OF THE MOdel}

Fig. 1 shows a typical oxide-confined VCSEL structure. It consists of an active region sandwiched between two distributed Bragg reflector (DBR) mirrors. These mirrors typically consist of 20-30 pairs of alternating low and high index material. In our model, the mirrors and the rest of the cavity are analyzed separately. That is, we first calculate the reflection properties of the top and bottom mirrors for a given wavelength. This information is then used as input for the cavity 


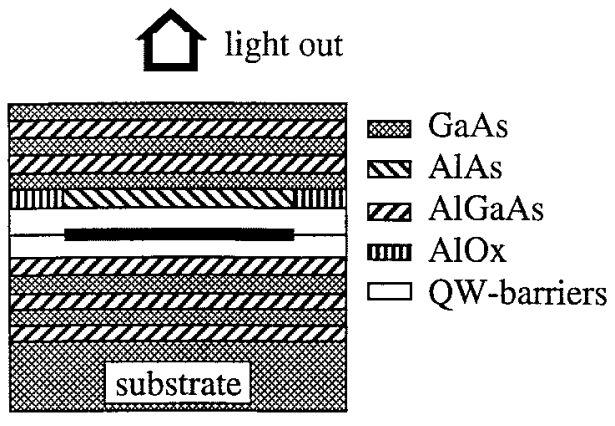

Fig. 1. VCSEL geometry after partial AlAs oxidation.

model, where we obtain an eigenvalue problem by taking into account propagation and interface effects in the quantum well (QW) and the cladding layers. The operating wavelength and material gain are subsequently adjusted in order to find an eigenvalue of one, in which case we have located a laser mode.

All calculations are based on Maxwell's equations assuming a time dependence given by $\exp (-j \omega t)$, with $\omega$ a real angular frequency. The direction of epitaxial growth, parallel to the propagation direction of the laser beam, will be referred to as the longitudinal or $z$ direction. Perpendicular to this direction, in the lateral plane, we define cylindrical coordinates $(r, \varphi)$. We only deal with VCSEL's that have a perfect rotational symmetry and a given refractive index profile for all the layers, including a given gain profile in the active region.

\section{A. Calculation of Mirror Properties}

VCSEL mirrors are traditionally layered structures. A general model for such mirrors has to be able to handle large refractive index contrasts, both in the lateral and the longitudinal direction. Moreover, in the presence of gain or absorption, complex refractive indices need to be considered. The combination of all these effects can lead to a complicated diffraction problem, where special care has to be taken not to compromise the numerical stability of the algorithm. The details of our model have been described in [15], but for the sake of clarity we will shortly outline the main points again.

First, consider a perfectly conducting metal cylinder of radius $R_{w}$, filled with a uniform reference dielectric. The eigenmodes of such a structure are well known and can be described in terms of Bessel functions. These functions form a complete orthogonal set that can be used to describe the different field components within the cylinder for any arbitrary dielectric profile and any excitation. Note that the reference dielectric does not need to be one that physically occurs in the structure under study. The sole purpose of the cylinder and the reference dielectric is to get a discrete set of eigenmodes, which we will call the "basic modes." We only retain the first $N$ basic modes to describe any field. The numerical influence of this metal cylinder is further discussed in Section III-D.

Now, we consider the actual structure under study, but placed within the metal cylinder of radius $R_{w}$. We first treat each layer (i.e., each lateral refractive index profile) separately, and we look for the modes that can propagate in such a layer. For each so-called "layer mode," we assume a $z$ dependence of the form $\exp (j q z)$, with $q$ the propagation

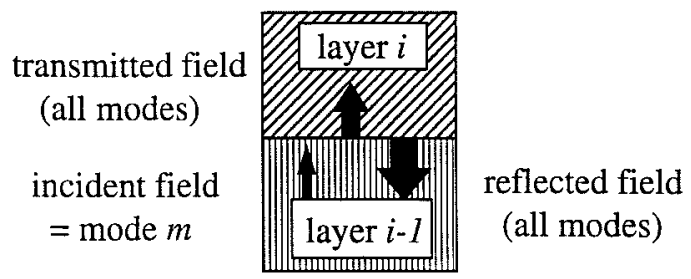

Fig. 2. Interface between layers $i-1$ and $i$ in a DBR mirror.

constant of the layer mode. These layer modes are expressed as an unknown linear combination of the basic modes. This representation is substituted into Maxwell's equations. The orthogonality of the basic modes reduces the expressions to an algebraic $N \times N$ eigenvalue problem that can be handled with standard numerical procedures. The eigenvalues of this problem correspond to the propagation constants of the layer modes and the corresponding eigenvectors give the field radial profiles of the layer mode.

It should be noted that in realistic mirrors most layers are uniform without any lateral index steps. In these layers, the modes are well known too, so in this case we do not have to go through the time-consuming process of solving the above eigenvalue problem.

Now that we know the $N$ layer modes, we can express any field in this layer as a superposition of layer modes propagating in the positive and in the negative $z$ direction. Thus, for the field in the $i$ th layer, we have

$$
\begin{aligned}
\mathbf{E}_{i}^{\text {tot }}(r, \varphi, z, t)= & \exp (-j \omega t) \sum_{k}\left\{A_{i, k}^{+} \mathbf{E}_{i, k}(r, \varphi) \exp \left(j q_{i, k} z\right)\right. \\
& \left.+A_{i, k}^{-} \mathbf{E}_{i, k}(r, \varphi) \exp \left(-j q_{i, k} z\right)\right\} \\
\mathbf{H}_{i}^{\text {tot }}(r, \varphi, z, t)= & \exp (-j \omega t) \sum_{k}\left\{A_{i, k}^{+} \mathbf{H}_{i, k}(r, \varphi) \exp \left(j q_{i, k} z\right)\right. \\
& \left.-A_{i, k}^{-} \mathbf{H}_{i, k}(r, \varphi) \exp \left(-j q_{i, k} z\right)\right\}
\end{aligned}
$$

in which $\mathbf{E}_{i, k}(r, \varphi)$ and $\mathbf{H}_{i, k}(r, \varphi)$ are the modal field profiles in the lateral plane of the $k$ th mode in the $i$ th layer. The propagation constant in the positive $z$ direction is given by $q_{i, k}$. All these quantities are known once the eigenvalue problem in the $i$ th layer is solved. The values of the $A_{i, k}$ coefficients follow from the excitation of each mode by a field incident upon the $i$ th layer.

Finally, we need to calculate the reflection and transmission at an interface between two layers. Consider Fig. 2 where the $m$ th mode of layer $i-1$ is incident on the interface between layers $i-1$ and $i$. This incident field will generate a reflected field in layer $i-1$ and a transmitted field in layer $i$. Thus, the field in layer $i-1$ will be of the form (1) with $A_{i-1, k}^{+}=\delta_{k m}$ and with the $A_{i-1, k}^{-}$coefficients having the meaning of reflection coefficients. In layer $i$, the field will only consist of modes propagating in the positive $z$ direction with the $A_{i, k}^{+}$coefficients having the meaning of transmission coefficients. The boundary conditions imposed by Maxwell's equations demand that the tangential field components are continuous across the interface. This holds for both the electric and magnetic fields. Repeating this for all possible incident modes of layer $i-1$ (i.e., $m=1 \ldots N$ ) and using the orthogonality relations for the modes of layer $i-1$, we obtain 


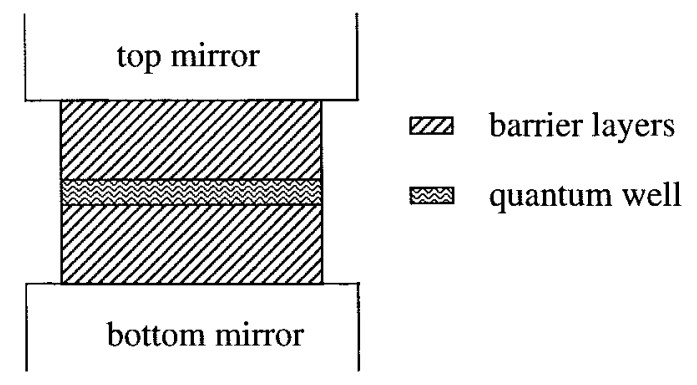

Fig. 3. Detailed view of the active region of a VCSEL.

an algebraic system of $2 N$ equations in $2 N$ unknowns that can be solved using standard numerical techniques. Solving this system, we find a $N \times N$ reflection matrix and a $N \times N$ transmission matrix that describe the reflected and transmitted fields for an arbitrary field incident from layer $i-1$. Reversing the role of layers $i$ and $i-1$ and repeating the whole procedure gives us another reflection and transmission matrix, this time for fields incident on the interface from layer $i$. These four matrices completely characterize the interface between the two layers for any incident field.

When describing an interface with a layer with lateral index steps, these matrices are completely filled, indicating that a certain amount of mode mixing occurs when light crosses that particular interface. This expresses scattering and diffraction. At interfaces between two uniform layers, all reflection and transmission matrices are diagonal, indicating that no scattering occurs and no diffraction other than the spreading of the beam while propagating.

Once all reflection and transmission matrices for all interfaces in the mirror are known, we can set up a recursive procedure (the so-called $R$-matrix scheme) to find the reflection and transmission properties of the complete mirror stack [16]. This scheme is numerically stable against large longitudinal refractive index changes.

From now on we will assume that the mirror reflection characteristics are known. We will refer to the reflection of the top mirror for fields coming from the active region as $\mathbf{R}^{\text {top }}$ and to the reflection of the bottom mirror for fields coming form the active region as $R^{\text {bot }}$.

The procedure outlined above is very general and able to treat complicated mirror structures. It can cope with all of the numerical problems outlined in the beginning of this section. Indeed, the eigenvalue problem for finding the layer modes is not fundamentally different for complex refractive indices as compared to real ones. Moreover, the techniques are stable against large index contrasts, both in the lateral and longitudinal directions.

\section{B. The Active Region and Formulation of the Cavity Problem}

In order not to complicate the mathematical formulation, we will restrict ourselves to an active region consisting of three layers: a QW between two barrier (cladding) layers. This situation is shown in Fig. 3. These layers can be either uniform or nonuniform.

Again, we look for all modes in the barrier layers and in the active layer, using the same method as explained in Section
II-A. Thus, in all three layers, (1) holds for the total field. In (1), we introduce the following notational convention:

$$
\begin{aligned}
& A_{\mathrm{bl}, k}^{+} \exp \left(j q_{\mathrm{bl}, k} z\right) \rightarrow A_{\mathrm{bl}, k}^{+}(z) \\
& A_{\mathrm{bl}, k}^{-} \exp \left(j q_{\mathrm{bl}, k} z\right) \rightarrow A_{\mathrm{bl}, k}^{-}(z)
\end{aligned}
$$

and analogous relations for the other $A$ coefficients.

At the interfaces between the barrier layers and the active layer, the tangential field components of both the electric and magnetic fields have to be continuous. (Since we work in the absence of any sources, this is equivalent to imposing the continuity of the normal components.) This leads to the following system of equations:

$$
\begin{gathered}
\sum_{k}\left\{A_{\mathrm{act}, k}^{+}\left(z_{1}\right) \mathbf{E}_{\mathrm{act}, k}^{t}(r, \varphi)+A_{\mathrm{act}, k}^{-}\left(z_{1}\right) \mathbf{E}_{\mathrm{act}, k}^{t}(r, \varphi)\right\} \\
=\sum_{k}\left\{A_{\mathrm{bl}, k}^{+}\left(z_{1}\right) \mathbf{E}_{\mathrm{bl}, k}^{t}(r, \varphi)+A_{\mathrm{bl}, k}^{-}\left(z_{1}\right) \mathbf{E}_{\mathrm{bl}, k}^{t}(r, \varphi)\right\} \\
\sum_{k}\left\{A_{\mathrm{act}, k}^{+}\left(z_{1}\right) \mathbf{H}_{\mathrm{act}, k}^{t}(r, \varphi)-A_{\mathrm{act}, k}^{-}\left(z_{1}\right) \mathbf{H}_{\mathrm{act}, k}^{t}(r, \varphi)\right\} \\
=\sum_{k}\left\{A_{\mathrm{bl}, k}^{+}\left(z_{1}\right) \mathbf{H}_{\mathrm{bl}, k}^{t}(r, \varphi)-A_{\mathrm{bl}, k}^{-}\left(z_{1}\right) \mathbf{H}_{\mathrm{bl}, k}^{t}(r, \varphi)\right\}
\end{gathered}
$$

in which the subscript act refers to the active layer, bl refers to the top barrier layer, the superscript $t$ refers to the tangential part of the fields, and $z_{1}$ is the $z$ position of the interface between the top barrier layer and the active layer (Fig. 3). Analogous equations hold for the interface between the bottom barrier layer and the active layer. Taking the vectorial product of (3) with $H_{\mathrm{act}, m}^{t}$ and applying the orthogonality relations leads to

$$
\begin{aligned}
& A_{\text {act }, m}^{+}\left(z_{1}\right)+A_{\text {act }, m}^{-}\left(z_{1}\right) \\
& \quad=\sum_{k} \mathbf{K}_{1, m k}\left\{A_{b 1, m}^{+}\left(z_{1}\right)+A_{b 1, m}^{-}\left(z_{1}\right)\right\} \\
& A_{\text {act }, m}^{+}\left(z_{1}\right)-A_{\text {act }, m}^{-}\left(z_{1}\right) \\
& \quad=\sum_{k} \mathbf{K}_{2, m k}\left\{A_{b 1, m}^{+}\left(z_{1}\right)-A_{b 1, m}^{-}\left(z_{1}\right)\right\} \\
& A_{\text {act }, m}^{+}\left(z_{2}\right)+A_{\text {act }, m}^{-}\left(z_{2}\right) \\
& \quad=\sum_{k} \mathbf{L}_{1, m k}\left\{A_{b 2, m}^{+}\left(z_{2}\right)+A_{b 2, m}^{-}\left(z_{2}\right)\right\} \\
& A_{\text {act }, m}^{+}\left(z_{2}\right)-A_{\text {act }, m}^{-}\left(z_{2}\right) \\
& \quad=\sum_{k} \mathbf{L}_{2, m k}\left\{A_{b 2, m}^{+}\left(z_{2}\right)-A_{b 2, m}^{-}\left(z_{2}\right)\right\}
\end{aligned}
$$

in which the following overlap integrals have been used:

$$
\begin{aligned}
\mathbf{K}_{1, m k}= & \left(\int d S \mathbf{u}_{z} \cdot \mathbf{E}_{\mathrm{bl}, k}^{t}(r, \varphi) \times \mathbf{H}_{\mathrm{act}, m}^{t}(r, \varphi)\right) \\
& \cdot\left(\int d S \mathbf{u}_{z} \cdot \mathbf{E}_{\mathrm{act}, m}^{t}(r, \varphi) \times \mathbf{H}_{\mathrm{act}, m}^{t}(r, \varphi)\right)^{-1} \\
\mathbf{K}_{2, m k}= & \left(\int d S \mathbf{u}_{z} \cdot \mathbf{E}_{\mathrm{act}, m}^{t}(r, \varphi) \times \mathbf{H}_{\mathrm{bl}, k}^{t}(r, \varphi)\right) \\
& \cdot\left(\int d S \mathbf{u}_{z} \cdot \mathbf{E}_{\mathrm{act}, m}^{t}(r, \varphi) \times \mathbf{H}_{\mathrm{act}, m}^{t}(r, \varphi)\right)^{-1} .
\end{aligned}
$$


Analogous relations hold for the $\mathbf{L}$ overlap matrices for the fields of the lower barrier layer. In (5), $\mathbf{u}_{z}$ is a unit vector in the positive $z$ direction and the surface integrals run over the entire area enclosed by the perfectly conducting cylinder.

Now that we have expressed the boundary conditions at the interfaces between the active layer and the barrier layers, we have to express the boundary conditions imposed by the mirrors. In matrix notation, these are

$$
\begin{aligned}
& {\left[A_{b 1}^{-}\left(z_{1}\right)\right]=\left[\exp \left(j q_{b 1} d_{b 1}\right)\right] \mathbf{R}^{\mathrm{top}}\left[\exp \left(j q_{b 1} d_{b 1}\right)\right]\left[A_{b 1}^{+}\left(z_{1}\right)\right]} \\
& {\left[A_{b 2}^{+}\left(z_{2}\right)\right]=\left[\exp \left(j q_{b 2} d_{b 2}\right)\right] \mathbf{R}^{\mathrm{bot}}\left[\exp \left(j q_{b 2} d_{b 2}\right)\right]\left[A_{b 2}^{-}\left(z_{2}\right)\right] .}
\end{aligned}
$$

Equations (6) also take into account propagation across the barrier layers over the respective distances $d_{b 1}$ and $d_{b 2}$. If we choose the origin of the $z$ axis in the center of the active layer, as done in Fig. 3, then we can also write

$$
\begin{aligned}
& A_{\text {act }, k}^{+}\left(z_{1}\right)=A_{\text {act }, k}^{+}(0) \exp \left(j q_{\text {act }, k} d_{\text {act }} / 2\right) \\
& A_{\text {act }, k}\left(z_{1}\right)=A_{\text {act }, k}^{-}(0) \exp \left(-j q_{\text {act }, k} d_{\text {act }} / 2\right) \\
& A_{\text {act }, k}^{-}\left(z_{2}\right)=A_{\text {act }, k}^{-}(0) \exp \left(j q_{\text {act }, k} d_{\text {act }} / 2\right) \\
& A_{\text {act }, k}^{+}\left(z_{2}\right)=A_{\text {act }, k}^{+}(0) \exp \left(-j q_{\text {act }, k} d_{\text {act }} / 2\right) .
\end{aligned}
$$

Taking the first and the second equation in (4) and the first one in (6), we can eliminate all $A$ coefficients relating to the top barrier layer. When we use (7) as well, we obtain a relation of the form

$$
A_{\text {act }, k}^{-}(0)=\sum_{i} \mathbf{C}_{1, k i} A_{\text {act }, i}^{+}(0)
$$

which relates the upward propagating fields in the center of the active layer to the downward propagating fields at the same $z$ position. Taking the remaining equations in (4) and (6), eliminating the $A$ coefficients relating to the lower barrier layer, and taking into account (7), we also find

$$
A_{\text {act }, k}^{+}(0)=\sum_{i} \mathbf{C}_{2, k i} A_{\text {act }, i}^{-}(0) .
$$

In matrix notation, (8) and (9) can be restated as

$$
\begin{aligned}
& {\left[A_{\text {act }}^{-}(0)\right]=\mathbf{C}_{1}\left[A_{\text {act }}^{+}(0)\right]} \\
& {\left[A_{\text {act }}^{+}(0)\right]=\mathbf{C}_{2}\left[A_{\text {act }}^{-}(0)\right] .}
\end{aligned}
$$

After eliminating the $A^{-}$coefficients, we get the following homogeneous system of $N$ equations that has to be solved for a nonzero solution:

$$
\left(\mathbf{C}_{2} \mathbf{C}_{1}-1\right)\left[A_{\text {act }}^{+}(0)\right]=0
$$

with 1 the unit matrix. We can only find nontrivial solutions if the determinant of the matrix $\mathbf{C}_{2} \mathbf{C}_{1}-\mathbf{1}$ vanishes. Since the mirrors are not perfectly reflecting, this determinant will not vanish for any real angular frequency $\omega$ unless we include gain in the active layer to overcome the loss. Another way of interpreting (11) is that we are looking for an eigenvector of the matrix $\mathbf{C}=\mathbf{C}_{2} \mathbf{C}_{1}$ that has an eigenvalue equal to 1 . This point of view was already formulated and used in [17].

\section{Numerical Procedure}

Equation (11) is ideally suited to be handled by the singular value decomposition (SVD) technique. If we are looking for the material gain needed to reach threshold, then the matrix $\mathbf{C}_{2} \mathbf{C}_{1}-\mathbf{1}$ becomes singular and should therefore be handled with care. SVD is a standard method to deal with singular algebraic problems. It returns a product decomposition of the input matrix of the following form:

$$
\mathbf{C}_{2} \mathbf{C}_{1}-\mathbf{1}=\mathbf{U} \Sigma \mathbf{V}^{H}
$$

in which $\mathbf{U}$ and $\mathbf{V}$ are unitary matrices, the superscript $H$ stands for Hermitian conjugate, and $\Sigma$ is a real positive diagonal matrix. These positive real numbers are called "singular values" of the original matrix. The columns of $\mathbf{V}^{H}$ that correspond to singular values equal to 0 are an orthonormal base of the nullspace of the original matrix and are therefore solutions to (11). Given (12), it is easy to see that the following holds:

$$
\begin{aligned}
\Sigma & =\left(\begin{array}{cccc}
\sigma_{1} & 0 & 0 \\
0 & \sigma_{2} & & \\
& & 0 & 0 \\
0 & & 0 & \sigma_{N}
\end{array}\right) \\
& =\left(\begin{array}{cccc}
\left|\gamma_{1}-1\right| & 0 & 0 \\
0 & \left|\gamma_{2}-1\right| & & 0 \\
0 & & 0 & \left|\gamma_{N}-1\right|
\end{array}\right)
\end{aligned}
$$

with $\sigma_{i}$ the singular values of the matrix $\mathbf{C}_{2} \mathbf{C}_{1}-1$ and $\gamma_{i}$ the eigenvalues of the matrix $\mathbf{C}=\mathbf{C}_{2} \mathbf{C}_{1}$. In general, these eigenvalues are complex numbers indicating a deviation from resonance for both the phase and the amplitude of the field. Phase resonance can be obtained by changing the wavelength $\lambda$, while amplitude resonance is achieved by changing the material gain $g_{\text {mat }}$ of the active layer. We also see from (13) that if $\sigma_{i}=0$ then indeed $\gamma_{i}=1$, as expected.

We now derive some expressions that will make it easier to locate the zero for $\sigma_{i}$ in the $\left(\lambda, g_{\text {mat }}\right)$-space.

From the relationship between $\sigma_{i}$ and $\gamma_{i}$, assuming that the imaginary part of $\gamma_{i}$ is sufficiently small, the following holds:

$$
\begin{aligned}
\sigma_{i}^{2} & =\left|\gamma_{i}-1\right|^{2}=\left|\gamma_{i}\right|^{2}-2 \operatorname{Re}\left(\gamma_{i}\right)+1 \\
\operatorname{Re}\left(\gamma_{i}\right) & =\sqrt{\left|\gamma_{i}\right|^{2}-\left(\operatorname{Im}\left(\gamma_{i}\right)\right)^{2}} \cong\left|\gamma_{i}\right|-\frac{1}{2} \frac{\left(\operatorname{Im}\left(\gamma_{i}\right)\right)^{2}}{\left|\gamma_{i}\right|}
\end{aligned}
$$

We can therefore deduce that

$$
\sigma_{i}^{2}-\frac{\left(\operatorname{Im}\left(\gamma_{i}\right)\right)^{2}}{\left|\gamma_{i}\right|} \cong\left(\left|\gamma_{i}\right|-1\right)^{2}
$$

Close to a cavity resonance, $\operatorname{Im}\left(\gamma_{i}\right)$ is indeed sufficiently small and $\left|\gamma_{i}\right|$ is more or less constant when we change the gain. Therefore, (15) means that the relationship between $\operatorname{Im}\left(\gamma_{i}\right)$ and $\sigma_{i}$ is given by a hyperbola. Furthermore, close to phase resonance the relationship between the wavelength $\lambda$ and $\operatorname{Im}\left(\gamma_{i}\right)$ is linear as well, since the phase of the mirrors varies linearly within the stopband. Therefore, the relationship between $\lambda$ and $\sigma_{i}$ is also given by a hyperbola.

This is an important conclusion. A typical $\left(\lambda, \sigma_{i}\right)$-plot for different values of $g_{\text {mat }}$ is shown in Fig. 4. For the lowest 


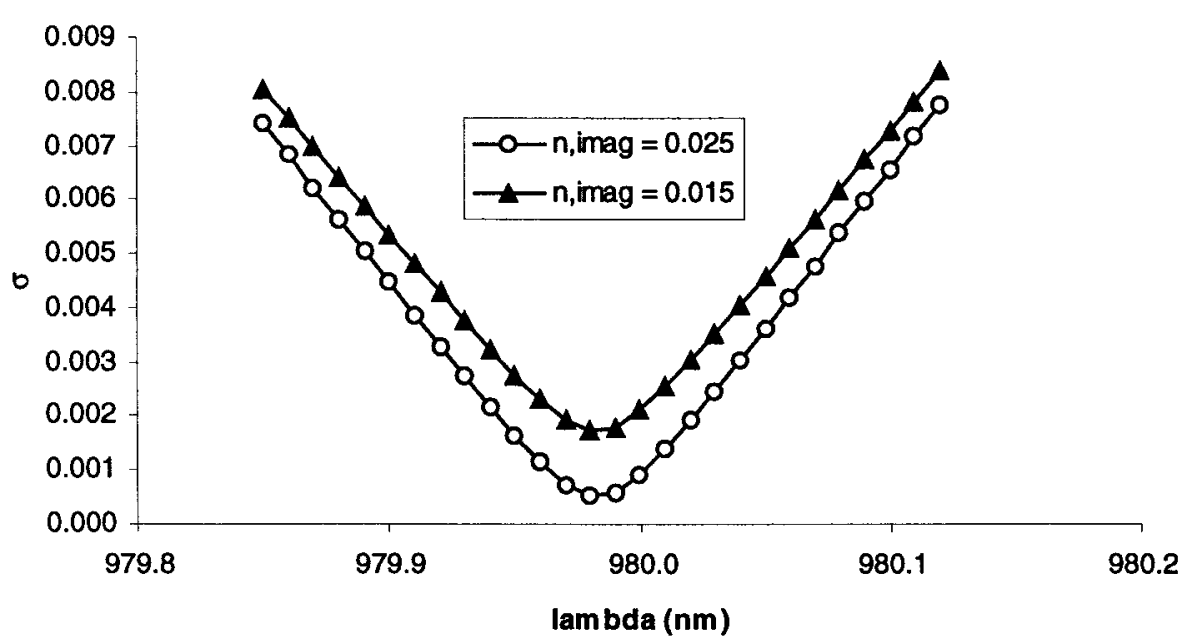

Fig. 4. Typical observed $\left(\lambda, \sigma_{i}\right)$-plot.

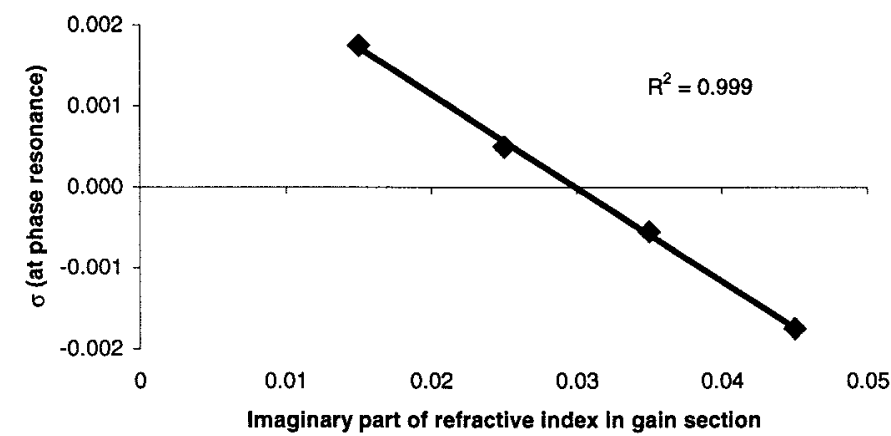

Fig. 5. Illustration of linear relationship at resonance between $\sigma_{i}$ and the material gain.

order laser modes, the hyperbola is usually nearly degenerate into two straight lines (the asymptotes of the hyperbola) and the curvature is only noticeable when phase resonance is quite strictly fulfilled. For the higher order laser modes, we find a hyperbola that is not degenerate.

Another important conclusion can be found when writing down the laser condition, assuming phase resonance is fulfilled, and close to amplitude resonance

$$
\left|R_{\text {mod }}^{\mathrm{top}} \cdot R_{\mathrm{mod}}^{\mathrm{bot}}\right| \exp \left(\frac{2 d_{\mathrm{act}}\left(g_{\mathrm{mat}} / 2\right) \Gamma_{\mathrm{mod}}}{\lambda}\right)=\gamma_{i} \cong 1 \text {. }
$$

In this expression, the factors in front of the exponential are the magnitudes of the amplitude reflectivities of both mirrors as experienced by the laser mode. The active region provides a power material gain $g_{\text {mat }}$ and has a thickness $d_{\text {act }}$. $\Gamma_{\text {mod }}$ is the lateral confinement of the laser mode within the gain section of the active layer. Since the argument of the exponential is very small, we can use a linear approximation leading to

$$
\sigma_{i}=|| R_{\mathrm{mod}}^{\mathrm{top}} \cdot R_{\mathrm{mod}}^{\mathrm{bot}}\left|\cdot\left(1+\frac{d_{\mathrm{act}} g_{\mathrm{mat}} \Gamma_{\mathrm{mod}}}{\lambda}\right)-1\right| .
$$

This implies that at phase resonance there is a linear relationship between $\sigma_{i}$ and $g_{\text {mat }}$, apart from the absolute value on the right-hand side of (17). This is the second important feature that helps us to locate the laser mode. It is illustrated in Fig. 5. The dots are the calculated minima of the hyperbolae

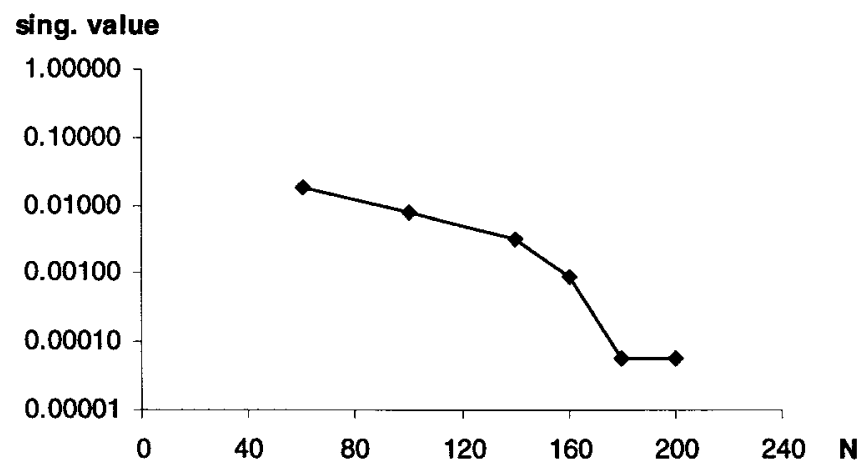

Fig. 6. Evolution of singular value as a function of number of basic modes.

for different values of the material gain. The straight line is obtained using a least squares fit to these points. In all cases the correlation coefficient $R^{2}$ is above $99 \%$, indicating a very good linear relationship between $\sigma_{i}$ and $g_{\text {mat }}$.

Thus, the algorithm used to identify a laser mode can be outlined as follows.

1) For different values of $g_{\text {mat }}$, adjust the wavelength to obtain phase resonance. While doing this, the $\left(\lambda, \sigma_{i}\right)$ plot looks like a hyperbola.

2) Plot the minima of the different $\left(\lambda, \sigma_{i}\right)$-plots (one minimum for every value of $\left.g_{\text {mat }}\right)$. After a change of sign for some values of $\sigma_{i}$, we obtain a straight line $\left(g_{\text {mat }}, \sigma_{i}\right)$.

3) The intercept of this straight line with the $g_{\text {mat }}$-axis yields the threshold material gain for that particular laser mode, since at that value of $g_{\text {mat }}$ we fulfill the condition $\sigma_{i}=0$.

\section{Convergence Analysis}

Finally, we investigate the number of basic modes that is required in order to get an accurate description of the fields. For our modal expansion, we retain the $N / 2$ lowest order TE modes of the reference cylinder and the $N / 2$ lowest order TM modes. Simultaneously using both TE and TM fields allows us deal with hybrid modes. Fig. 6 shows, as a function of the total number $N$ of basic modes, the evolution of the singular value $\sigma_{i}$, calculated at the laser resonance $\left(\lambda_{200}, g_{\text {mat,200 }}\right)$ found 


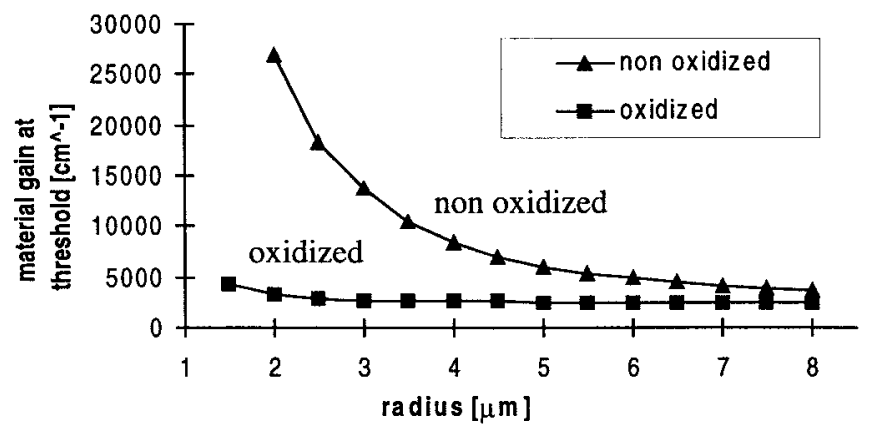

Fig. 7. Threshold material gain for the fundamental laser mode.

using $N=200$. It can be seen that around $N=180$ a stable situation is reached with a singular value that is sufficiently close to zero. All the other examples in this paper are therefore calculated with this number of basic modes. It should be noted that, for this value of $N$, around 10 to 15 basic modes are evanescent. It is well known that one should retain a number of evanescent modes that is sufficiently high in order to have an accurate representation of the fields.

\section{Simulation Results}

\section{A. Comparison of Proton-Implanted and Oxidized VCSEL's}

Fig. 1 shows an oxide-confined top-emitting VCSEL. The bottom mirror is a 30-pair $\operatorname{GaAs}(n=3.525)$ $\mathrm{Al}_{0.8} \mathrm{Ga}_{0.2} \mathrm{As}(n=3.077)$ mirror. The active layer is a single 5-nm-thick QW embedded in a $\lambda$-cavity with $n=3.525$ everywhere. The top mirror is a 24-period $\operatorname{GaAs}(n=3.525)-\mathrm{Al}_{0.8} \mathrm{Ga}_{0.2} \mathrm{As}(n=3.077)$ mirror with one additional pair of layers $\operatorname{GaAs}(n=3.525)-\operatorname{AlAs}(n=2.952)$ right above the active region. This AlAs layer is oxidized to form an oxide window of variable radius $R$. The oxide confined VCSEL will be compared to a proton-implanted (nonoxidized) VCSEL that has an identical structure, except for the top mirror which is a 25-period GaAs $(n=3.525)-\mathrm{Al}_{0.8} \mathrm{Ga}_{0.2} \mathrm{As}(n=3.077) \mathrm{DBR}$. The radius of the gain section in the QW is the same in both structures and equals the radius of the oxide window in the oxidized VCSEL. Outside the gain region, the QW has a constant absorption $\left(n_{\mathrm{imag}}=-0.1\right)$. This absorption is included for physical reasons, but it also avoids finding dummy cavity resonances. The design wavelength for the fundamental laser mode is $980 \mathrm{~nm}$.

Fig. 7 shows the threshold material gain for the fundamental mode in both structures. (In interpreting these values, keep in mind that all calculations are done for a device with a single QW. A real VCSEL typically has three QW's, so the values in Fig. 7 should be divided by three in order to get realistic values for the required gain per $\mathrm{QW}$.) We can conclude from Fig. 7 that a proton implanted VCSEL with $R<3.5 \mu \mathrm{m}$ is difficult to realize, while oxide confined VCSEL's can be made much smaller than that [9]. Fig. 7 is therefore very much in agreement with experimental findings [18], [19].

We now show that this lower threshold for oxide-confined VCSEL's can be correlated with a better confinement of

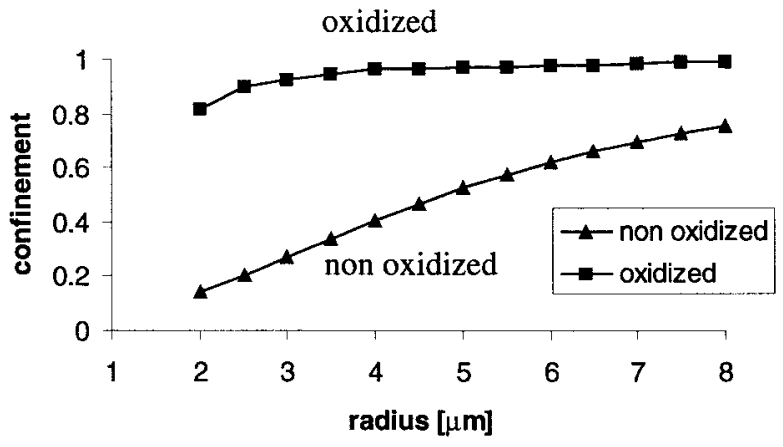

Fig. 8. Confinement of the fundamental laser mode in the gain section of the active layer.

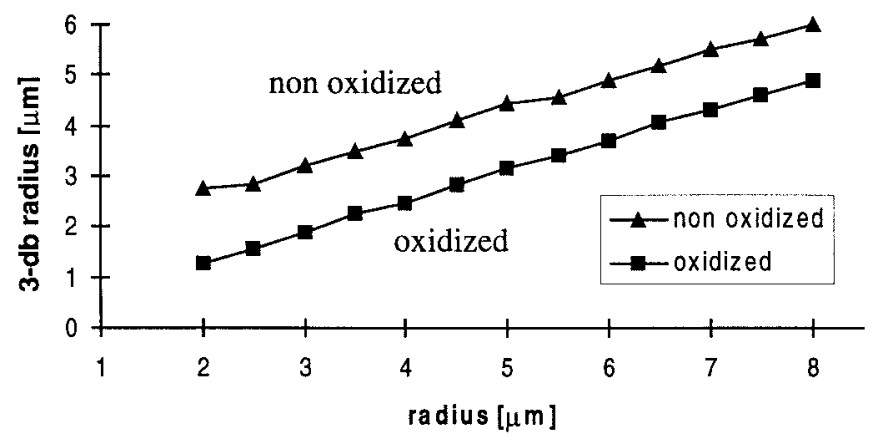

Fig. 9. The fundamental laser mode with 3-dB radius.

the laser mode to the gain region and with a higher modal reflectivity, counteracting diffraction losses.

Fig. 8 shows the confinement factor $\Gamma_{\text {mod }}$ of the laser mode within the gain section of the QW. For nonoxidized devices, $\Gamma_{\text {mod }}$ hardly reaches $75 \%$ when $R<8 \mu \mathrm{m}$. For very small VCSEL's of $2-\mu \mathrm{m}$ radius, the overlap even goes down to $14 \%$. This reduced overlap with the gain section means that the laser mode will not be pumped efficiently. The oxidation procedure leads to a vast increase in $\Gamma_{\text {mod }}$. For large oxidized devices, the overlap is between $95 \%$ and $99 \%$, going down to $82 \%$ for VCSEL's of $2-\mu \mathrm{m}$ radius. The material gain of the QW is therefore much more efficiently used in oxide-confined structures.

Instead of looking at the overlap $\Gamma_{\text {mod }}$, we can also plot the $3-\mathrm{dB}$ radius of the transverse field profile of the laser mode. Larger overlap translates evidently into narrower field profiles, as can be seen from Fig. 9. For a large range of diameters, the difference in $3-\mathrm{dB}$ radius is more or less constant at a value of $1.2 \mu \mathrm{m}$. When $R<2.5 \mu \mathrm{m}$, the field profile of the nonoxidized VCSEL's seems to widen again. This is an effect that has already been discussed by Ujihara [20] and can be understood based on the reflection characteristics of a uniform DBR mirror. If the laser mode were to become too narrow, it would contain many plane-wave contributions that propagate under very oblique angles and that are therefore not well reflected by the DBR mirror. These plane-wave components cannot contribute to the laser mode. This leads in effect to a lower bound on the laser mode $3-\mathrm{dB}$ radius.

Fig. 10 shows the product of top and bottom mirror amplitude reflectivity, which can be determined from (17), once we have obtained the threshold gain and the confinement factor. 


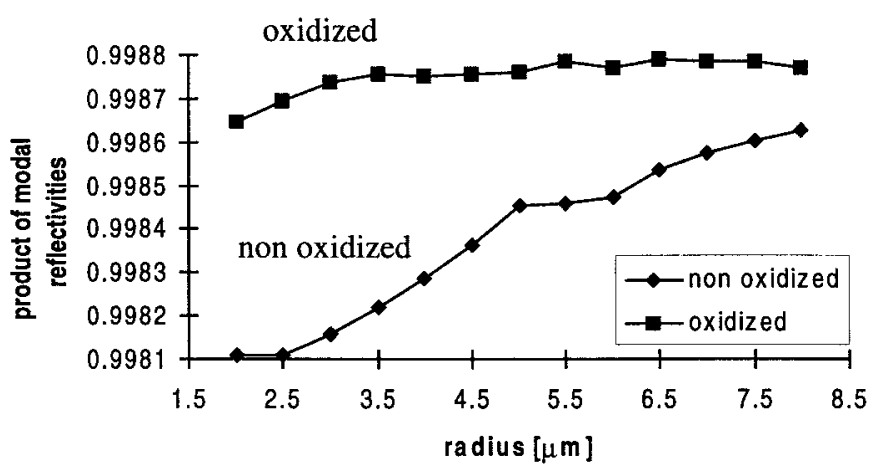

Fig. 10. Product of mirror reflectivities for the fundamental laser mode.

We see that in both cases this combined amplitude reflectivity is very high, always above $99.8 \%$. For both structures, the reflectivity goes down with decreasing device radius. This is consistent with the intuitive understanding that a narrow beam suffers more diffraction loss. However, the reflectivity for the oxide-confined structures is always higher than for the protonimplanted ones, meaning that the oxide is very effective in counteracting diffraction losses. Only for very small devices $(R<2 \mu \mathrm{m})$, diffraction loss becomes again a competing factor that cannot be completely neutralized anymore by the oxide aperture. In these cases, the threshold material gain will increase, as was already apparent from Fig. 7. Still, this increase is not as dramatic as compared to nonoxidized devices, because the overlap of the fundamental mode with the gain section is still very large (Fig. 8).

To summarize the effect of diffraction losses on VCSEL's we can say the following. In a proton-implanted device only the weak gain guiding counteracts diffraction losses. When reducing the device diameter, the laser mode will therefore be less confined to the gain section. This in turn leads to an increased threshold for laser action. In oxide-confined VCSEL's, the large index difference caused by the oxidation effectively counteracts diffraction when $R>3 \mu \mathrm{m}$, where the threshold material gain (Fig. 7) and the overlap with the gain section (Fig. 8) do not change significantly with oxide aperture radius. For smaller devices $(R<2 \mu \mathrm{m})$, diffraction again becomes an important effect. This leads a to reduced overlap of the laser mode with the gain section and an increased material gain needed to reach threshold, although these effects are not as pronounced as compared to proton-implanted devices.

\section{B. Effect of Oxide Position on Modal Stability}

In all of the above calculations, the thickness of the oxidized layer was kept fixed at $80 \mathrm{~nm}$, meaning that a complete quarter wavelength layer of semiconductor material was oxidized. We will now vary oxide thickness and position relative to the standing wave pattern in the cavity, since we expect that the degree at which the oxide is sensed by the laser mode will vary at different positions relative to the standing wave pattern. We consider two positions within the $\lambda / 4$ AlGaAs-layer: "antinode position" (Fig. 11) and "node position" (Fig. 12). Note, however, that these are not exactly antinode and node positions, because the oxidized AlAs layer has a nonzero thickness and is always kept within the $\lambda / 4$ AlGaAs-layer.

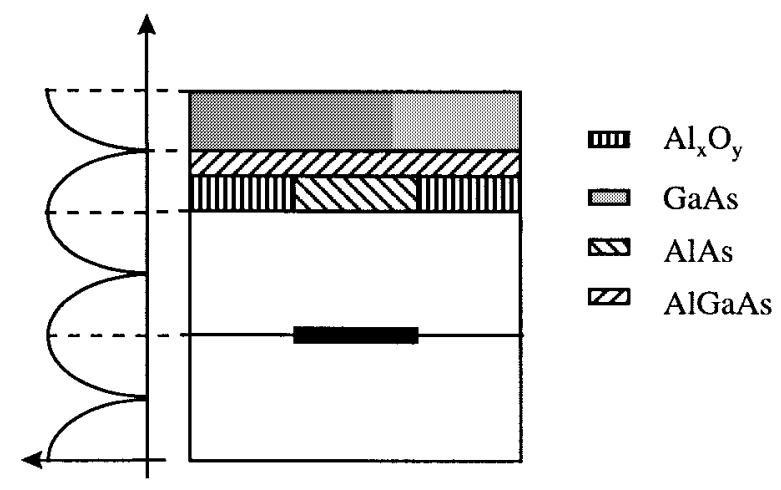

Fig. 11. Oxidized VCSEL with the oxide aperture at the antinode position.

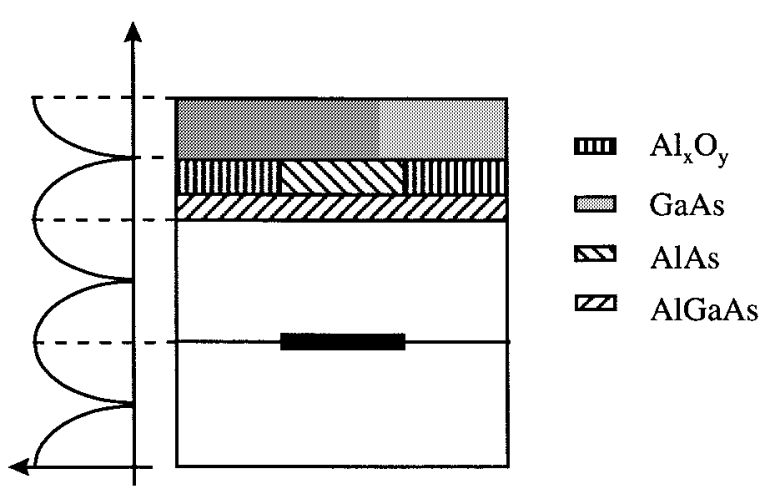

Fig. 12. Oxidized VCSEL with the oxide aperture at the node position.

In the "antinode position," the bottom of the oxidized layer is at the antinode position, while in the "node position" the top of this oxidized layer is at the node position.

It will become clear from the simulation results that an antinode oxide leads to a much reduced threshold gain. Indeed, the aperture is placed at a maximum of the standing wave pattern and is therefore very effective in counteracting diffraction. However, this is true for both the fundamental mode and the higher order modes. Therefore, an antinode oxide is able to provide for low threshold gain for the fundamental mode, but at the expense of a reduced modal selectivity.

In a node oxide, exactly the opposite is true. The aperture is placed at a field minimum and is not very effective in counteracting diffraction. These detrimental diffraction effects are worse for the higher order modes, and therefore a node oxide provides for a good modal stability, but at the expense of a higher threshold for the fundamental mode.

The difference between a node and an antinode oxide with regard to modal stability is illustrated in Fig. 13. This figure shows the difference in threshold gain for the fundamental mode and the first-order mode for $R=4 \mu \mathrm{m}$. Node oxides clearly reduced modal stability, i.e., smaller threshold difference between the modes.

The same conclusions can be drawn if we look at the overlap with the gain section. Fig. 14 shows that an antinode oxide confines the modes a lot better to the gain section than a node oxide. However, the difference in overlap for the fundamental and the first-order mode is larger for a node oxide than for an antinode oxide. 


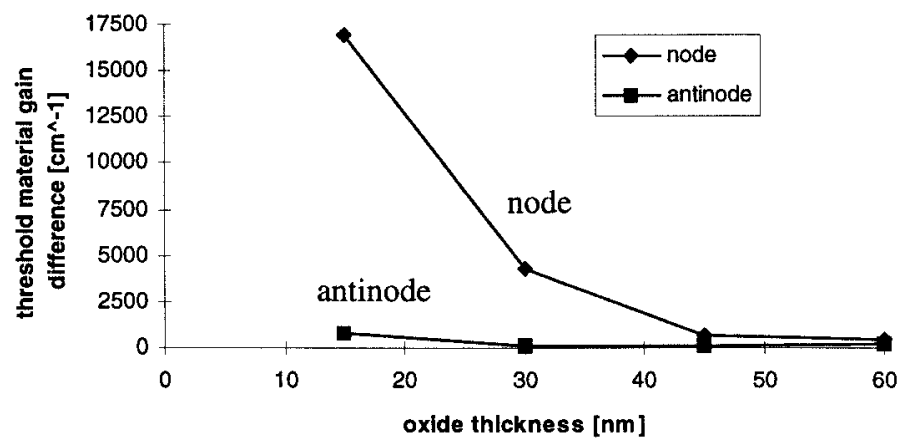

Fig. 13. Difference in threshold material gain for fundamental and first-order modes in oxidized VCSEL's.

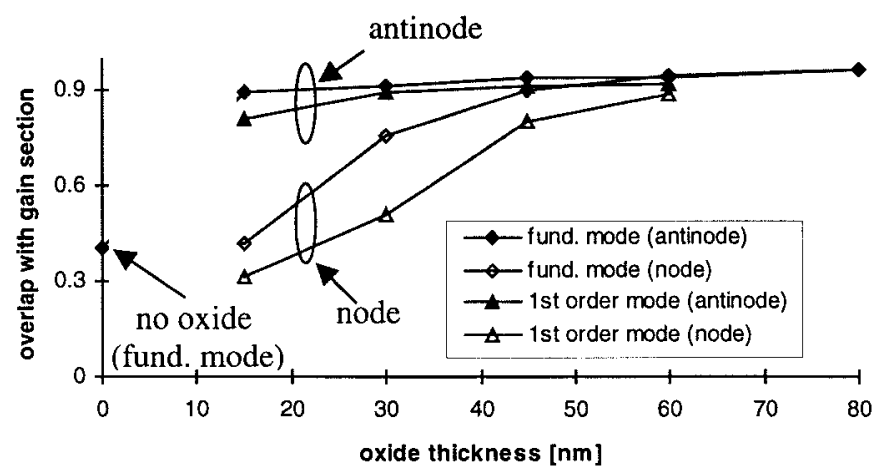

Fig. 14. Overlap of fundamental and first-order mode with gain section in oxide-confined VCSEL's (radius of the oxide aperture: $4 \mu \mathrm{m}$ ).

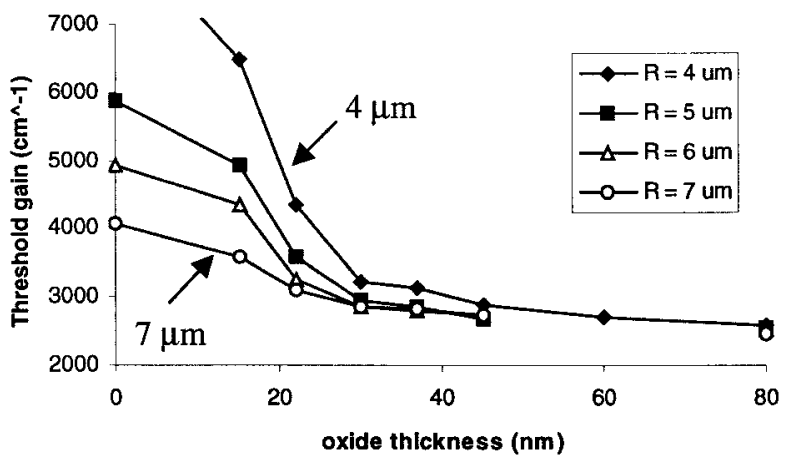

Fig. 15. Threshold material of the fundamental mode as a function of oxide thickness for various values of the aperture radius (node oxide).

We now investigate the modal stability properties of oxidized VCSEL's with $R>4 \mu \mathrm{m}$. Given the results presented above, we only consider node oxide windows. The threshold material gain for these devices is shown in Fig. 15 (fundamental mode) and in Fig. 16 (first-order mode). We can see, e.g., that, for aperture thicknesses of 30 and $20 \mathrm{~nm}$, the threshold material gain for the fundamental mode is at most $3200 \mathrm{~cm}^{-1}$, while for the first-order mode it is at least $4800 \mathrm{~cm}^{-1}$, if $R<6 \mu \mathrm{m}$. Therefore, in this range of parameters, we have an acceptable compromise between low threshold for the fundamental mode and good modal stability. Probably, a device with $R=7 \mu \mathrm{m}$ is too large to maintain a stable fundamental mode for any reasonable threshold for the fundamental mode.

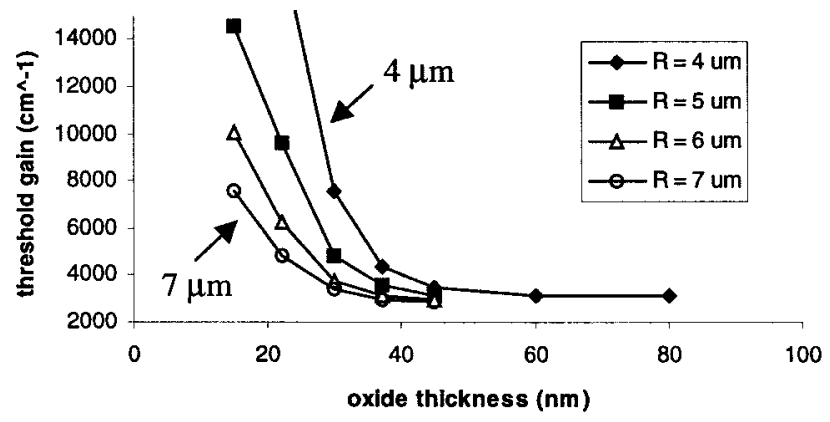

Fig. 16. Threshold material gain of the first-order mode as a function of oxide thickness for various values of the aperture radius (node oxide).

We can summarize this in the following rules of thumb for stable mode operation:

$$
\begin{aligned}
\Gamma_{0}-\Gamma_{1} & \geq 0.15 \\
\Gamma_{0} & \geq 0.75
\end{aligned}
$$

where $\Gamma_{0}$ stands for the overlap of the fundamental mode with the gain section and $\Gamma_{1}$ for the overlap of the first-order mode. The first condition assures a fairly large difference in threshold for both modes, while the second ensures that the threshold for the fundamental mode is acceptable.

We can expect that current spreading will slightly change both confinement factors, but their difference should remain more or less unaltered as compared to the values we find. The reason for this is that this process is not very mode-selective. Other thermal and electrical effects like current crowding, thermal lensing, and carrier-induced antiguiding could have a larger impact on modal stability. However, these effects could still be taken into account in our optical model by changing the uniform gain profile in the QW to a staircase approximation of the real gain profile resulting from these phenomena.

\section{Plane-Wave Model for Oxide Apertures}

The rigorous simulation in the previous section showed that an antinode oxide is better at confining the laser mode to the action region, leading to higher values of $\Gamma$. We can further clarify this by considering simple plane-wave calculations.

We calculate the plane-wave reflectivity for two situations. One is where we place an oxide layer of thickness 20, 40, or 60 $\mathrm{nm}$ at various distances from a 20-period GaAs-AlGaAs DBR. This situation corresponds to the tails of the laser mode that extend under the oxide window. The other situation is similar, but the oxide is replaced by an AlAs layer. This corresponds to the center part of the laser mode. This is done for distances between oxide and mirror between 0 and $160 \mathrm{~nm}$, but note that, in a real VCSEL with the oxide embedded in a $\lambda / 4$ AlAs-layer, the actual range is limited between 0 and $80 \mathrm{~nm}$.

Fig. 17 shows the difference in amplitude reflectivity for the two situations. We see that in the range $0-80 \mathrm{~nm}$, an oxide layer always has a lower mirror reflectivity than the central section. This implies that the tails of the field will suffer more mirror transmission loss than the central part, leading to a better confinement.

To compare node to antinode oxides, we remark that a node oxide of thickness $d_{\mathrm{ox}}$ corresponds to $d=0$ in Fig. 17, while 


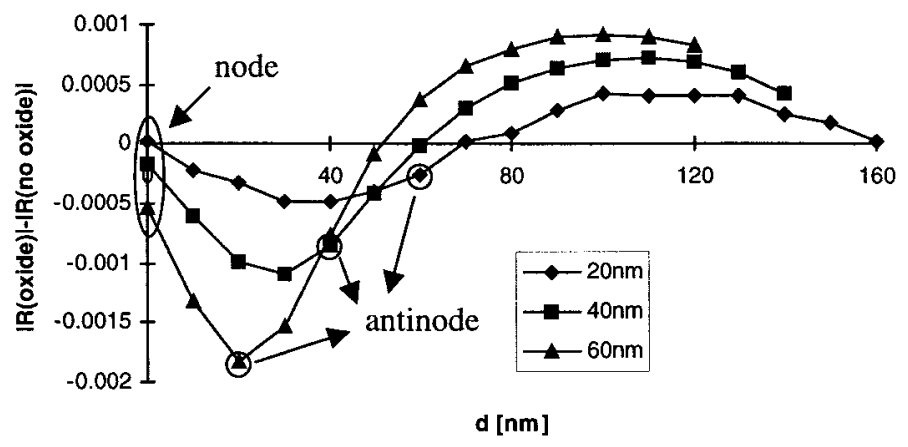

Fig. 17. Change in modulus of the mirror amplitude reflectivity due to the oxide as a function of the distance $(d)$ between the oxide and the DBR.

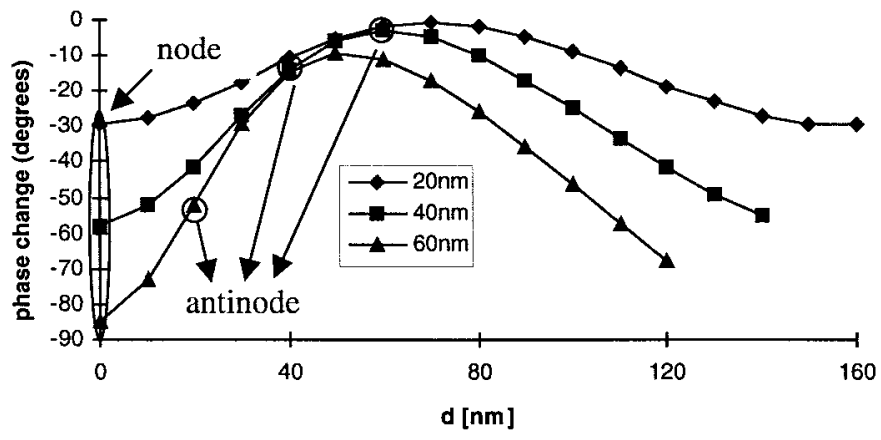

Fig. 18. Phase change of the mirror amplitude reflectivity due to the oxide as a function of the distance $(d)$ between the oxide and the DBR.

an antinode oxide of thickness $d_{\mathrm{ox}}$ corresponds to $d=80 \mathrm{~nm}$ $-d_{\mathrm{ox}}$. We see indeed that the additional mirror transmission loss suffered by the tails of the mode is higher for an antinode oxide than for a node oxide. Therefore, with antinode oxides, the modes are much better confined to the central region, minimizing mirror transmission loss.

Finally, the phase change as shown in Fig. 18 demonstrates that the oxide aperture acts as a focusing optical element, as already discussed in [9].

\section{Influence of the Metal Cylinder}

Lastly, we discuss the influence of the perfectly conducting wall of radius $R_{w}$ that was introduced for numerical reasons (see Section II-C). All results shown above were calculated with $R_{w}=12 \mu \mathrm{m}$. In principle, this metal wall introduces (numerical) reflections that can corrupt the results, because in a real structure this numerically reflected power would be radiated out of the laser. To test the influence of this effect, we can use two approaches. The first is to introduce mild absorbers between the structure of interest and the metallic wall. If we take care that the absorbers themselves do not introduce any parasitic reflections, we can make sure that the power is absorbed before it reaches the metal wall. A second approach is simply to place the metallic wall further away from the structure of interest.

Both methods were used to check the influence of the metal wall. For oxide-confined VCSEL's, we used radii of $R=2$, 4 , or $8 \mu \mathrm{m}$. For proton-implanted devices, we used $R=4$ or $8 \mu \mathrm{m}$.
First, we kept the metal wall at $R_{w}=12 \mu \mathrm{m}$ and introduced an absorber in the top mirror extending laterally from 10 to $12 \mu \mathrm{m}$. The imaginary part of the refractive index was chosen so that only $0.1 \%$ of the perpendicularly incident power was reflected at the absorber interface. The threshold material gain for the oxide-confined VCSEL's showed no noticeable difference with the results presented in Fig. 7. For the $4-\mu \mathrm{m}$ proton-implanted structure, we found agreement with Fig. 7 to within $2 \%$, while for the $8-\mu \mathrm{m}$ proton-implanted device deviations of $20 \%$ were found. For this reason, we only tried putting the metal cylinder further away for the $8-\mu \mathrm{m}$ proton-implanted VCSEL. When the metallic wall was put at $R_{w}=25 \mu \mathrm{m}$ without any absorbers present, we again found agreement with Fig. 7 to within $2 \%$.

Therefore, we can conclude that the results of Fig. 7 are correct to within $2 \%$, and that for the oxide-confined structures the presence of the metal wall is negligible, while for the nonoxidized structures, more care should be taken in assessing the accuracy of the results.

\section{CONCLUSION}

We presented a vectorial electromagnetic model capable of determining the optical properties of currently used VCSEL's. Specifically, the high lateral index contrast due to oxide windows can be treated in an exact way, contrary to other models available until now.

Using this model, we compared oxide-confined structures to proton-implanted ones in order to understand and quantify the different mechanisms active in these structures. We showed that mirror diffraction loss in oxide-confined devices is effectively counteracted by the aperture, leading to a better overlap with the active region and to a reduced threshold material gain.

We also compared node oxides to antinode oxides, indication that there is a tradeoff to make between low threshold for the fundamental mode and good modal stability. Node oxides are favorable for modal stability, while antinode oxides yield low threshold gain for the fundamental mode.

\section{REFERENCES}

[1] M. H. MacDougal, P. D. Dapkus, V. Pudikov, H. Zhao, and G. M. Yang, "Ultralow threshold current vertical cavity surface emitting lasers with AlAs oxide-GaAs distributed Bragg reflectors," IEEE Photon. Technol. Lett., vol. 7, pp. 229-231, Mar. 1995.

[2] Y. Hayashi, T. Mukaihara, N. Hatori, N. Ohnoki, A. Matsutani, F. Koyama, and K. Iga, "Lasing characteristics of low threshold oxide confinement InGaAs-GaAlAs vertical cavity surface emitting lasers," IEEE Photon. Technol. Lett., vol. 7, pp. 1234-1236, Nov. 1995.

[3] K. L. Lear, K. D. Choquette, R. P. Schneider, S. P. Kilcoyne, and K M. Geib, "Selectively oxidized vertical cavity surface emitting lasers with 50\% power conversion efficiency," Electron. Lett., vol. 31, no. 3, pp. 208-209, 1995

[4] K. D. Choquette, R. P. Schneider, K. L. Lear, and K. M. Geib, "Low threshold voltage vertical cavity lasers fabricated by selective oxidation," Electron. Lett., vol. 30, pp. 2043-2044, 1994.

[5] K. D. Choquette, K. L. Lear, R. P. Schneider, K. M. Geib, J. J. Figiel, and R. Hull, "Fabrication and performance of selectively oxidized vertical cavity lasers," IEEE Photon. Technol. Lett., vol. 7, pp. 1237-1239, Nov. 1995.

[6] K. J. Ebeling, U. Fiedler, R. Michalzik, G. Reiner, and B. Weigl, "Recent advances in semiconductor vertical cavity lasers for optical communications and optical interconnects," in Proc. 22nd Eur. Conf. Optical Communication (ECOC), 1996, paper TuC.2.1. 
[7] G. R. Hadley, "Effective index model for vertical cavity surface emitting lasers," Opt. Lett., vol. 20, no. 13, pp. 1483-1485, 1995.

[8] E. R. Hegblom, D. I. Babic, B. J. Thibeault, and L. A. Coldren, "Estimation of scattering losses in dielectrically apertured vertical cavity lasers," Appl. Phys. Lett., vol. 68, no. 13, pp. 1757-1759, 1996.

[9] L. A. Coldren, B. J. Thibeault, E. R. Hegblom, G. B. Thompson, and J. W. Scott, "Dielectric apertures as intracavity lenses in vertical cavity lasers," Appl. Phys. Lett., vol. 68, no. 3, pp. 313-315, 1996.

[10] G. R. Hadley, K. L. Lear, M. E. Warren, K. D. Choquette, J. W. Scott, and S. W. Corzine, "Comprehensive numerical modeling of verticalcavity surface-emitting lasers," IEEE J. Quantum Electron., vol. 32, pp. 607-616, Apr. 1996.

[11] G. R. Hadley, "Low-truncation-error finite difference equations for photonics simulation II: Vertical-cavity surface-emutting lasers," $J$. Lightwave Technol., vol. 16, pp. 142-151, Jan. 1998.

[12] M. J. Noble, J. P. Loehr, and J. A. Lott, "Analysis of microcavity lasing modes using a full-vector weighted index method," IEEE. J. Quantum Electron., vol. 34, pp. 1892-1903, Oct. 1998.

[13] D. Burak and R. Binder, "Cold-cavity vectorial eigenmodes of VCSEL's," IEEE J. Quantum Electron., vol. 33, pp. 1205-1215, July 1997.

[14] R. Kuszelewicz and G. Aubert, "Modal matrix theory for light propagation in laterally restricted stratified media," J. Opt. Soc. Amer. A, vol. 14, no. 12, pp. 3262-3272, 1997.

[15] B. Demeulenaere, D. D. Zutter, and R. Baets, "Rigorous electromagnetic study of diffraction loss in VCSEL mirrors," Proc. Inst. Elect. Eng., pt J, vol. 143 , no. 4, pp. 221-227, 1996.

[16] L. Li, "Multilayer modal method for diffraction gratings of arbitrary profile, depth and permittivity," J. Opt. Soc. Amer. A, vol. 10, no. 12 pp. 2581-2590, 1993.

[17] B. Demeulenaere, B. Dhoedt, S. Goeman, and R. Baets, "Accurate three dimensional modeling of transverse mode threshold gain in vertical cavity surface emitting lasers," in Proc. Integrated Photonics Research'96 $(I P R)$, 1996, paper ITuE4.
[18] F. Peters, G. Robinson, M. Peters, D. Young, and L. Coldren, "Small electrically pumped index guided vertical cavity lasers," IEEE Photon. Technol. Lett., vol. 6, pp. 1176-1178, Oct. 1994.

[19] T. Oh, D. Hufaker, and D. Deppe, "Size effects in small oxide confined vertical cavity surface emitting lasers," Appl. Phys. Lett., vol. 69, no. 21, pp. 3152-3154, 1996.

[20] K. Ujihara, "Spontaneous emission and the concept of effective area in a very short optical cavity with plane parallel dielectric mirrors," Jpn.. J. Appl. Phys., vol. 30, no. 5B, pp. L901-L903, 1991.

B. Demeulenaere, photograph and biography not available at the time of publication.

P. Bienstman, photograph and biography not available at the time of publication.

B. Dhoedt, photograph and biography not available at the time of publication.

R. G. Baets (M'88-SM'96), photograph and biography not available at the time of publication. 